\title{
Tumor innervation: peripheral nerves take control of the tumor microenvironment
}

\author{
Stefan M. Gysler ${ }^{1,2}$ and Ronny Drapkin ${ }^{1,2,3}$ \\ 'Department of Obstetrics and Gynecology, Division of Gynecologic Oncology, ${ }^{2}$ Penn Ovarian Cancer Research Center, Department of Obstetrics and Gynecology, and ${ }^{3}$ Basser Center for BRCA, Abramson \\ Cancer Center, University of Pennsylvania Perelman School of Medicine, Philadelphia, Pennsylvania, USA.
}

\begin{abstract}
In recent decades, cancer research has expanded exponentially beyond the study of abnormally dividing cells to include complex and extensive heterotypic interactions between cancer and noncancer cells that constitute the tumor microenvironment (TME). Modulation of stromal, immune, and endothelial cells by cancer cells promotes proliferation, survival, and metabolic changes that support tumor growth and metastasis. Recent evidence demonstrates that tumors can recruit peripheral nerves to the TME, leading to enhanced tumor growth in a range of cancer models through distinct mechanisms. This process, termed tumor innervation, is associated with an aggressive tumor phenotype and correlates with poor prognosis in clinical studies. Therefore, the peripheral nervous system may play an underrecognized role in cancer development, harboring targetable pathways that warrant investigation. To date, nerves have been implicated in driving proliferation, invasion, metastasis, and immune evasion through locally delivered neurotransmitters. However, emerging evidence suggests that cell-cell communication via exosomes induces tumor innervation, and thus exosomes may also mediate neural regulation of the TME. In this Review, seminal studies establishing tumor innervation are discussed, and known and putative signaling mechanisms between peripheral nerves and components of the TME are explored as a means to identify potential opportunities for therapeutic intervention.
\end{abstract}

\section{Introduction}

Twenty-years ago, Hanahan and Weinberg distilled the antecedent decades of cancer research into a set of six "hallmarks of cancer," predicting a necessary shift in focus from viewing cancers as a mass of abnormally proliferating cells toward the consideration of tumors as neo-organs, with complex interactions between numerous cell types and organ systems that contribute to the malignant potential of a cancer $(1,2)$. Since the delineation of the hallmarks of cancer, a wealth of new evidence has emerged describing complex heterotypic interactions between cancer and noncancer cells that constitute a tumor, collectively known as the tumor microenvironment (TME).

The TME is composed of stromal cells, vasculature, immune cells, and a milieu of signaling molecules within an extracellular matrix (ECM). Although its composition is heterogeneous, the TME serves similar biological roles across multiple cancers related to metabolic support, angiogenesis, metastasis, chemoresistance, and, importantly, immune regulation. As each of these processes is critical to the development and progression of solid tumors, numerous clinical treatments have been developed to target elements of the TME, with varying degrees of success.

For example, the discovery of increased VEGF and immune checkpoint protein expression in solid tumors has led to the development of specific antibody treatments aimed at halting angiogenesis

Conflict of interest: RD is an advisory board member for Repare Therapeutics and VOC Health.

Copyright: () 2021, American Society for Clinical Investigation.

Reference information: J Clin Invest. 2021;131(11):e147276.

https://doi.org/10.1172/JCI147276. and enhancing antitumor immunity. While clinical benefits have been observed from these treatments alone or in combination with traditional chemotherapies, the results have been disparate among cancer types, and durable responses remain elusive (3-6).

Taken together, the evidence heretofore sheds light on the complexities of the TME and the difficulties in identifying actionable targets for treatment. While the partial successes of these therapies are promising, multiple redundant or alternative mechanisms likely exist to promote tumor progression even in the face of antiangiogenic or immunobiologic agents. Indeed, the efficacy of these medications is improved in many trials when combined with other agents (7). Thus, there remains a dire need for further investigation into the TME for alternative targets that can be leveraged to enhance or replace current treatments.

Interestingly, the role of the nervous system in solid tumor progression has been largely ignored until recently. While nerve fibers were noted in several cancers as early as in the 1900s $(8,9)$, further research focused primarily on perineural invasion (PNI), the process by which tumors invade existing locoregional nerves, leading to metastasis and reciprocal tumor and nerve growth (10). PNI has since been described in a range of solid tumors and correlates with aggressive tumor phenotypes and poor patient survival $(11,12)$. Despite this clinical relevance, relatively few investigators have studied the functional role of nerves in carcinogenesis and regulation of the TME. Conceptually, however, the nervous system stands to play an important role in influencing cancer biology from the earliest stages. First, nerves and blood vessels develop in parallel throughout the body during development, and multiple angiogenic factors have been shown to affect neuronal growth and vice versa. Moreover, several distinct pathways 
exist allowing for crosstalk between nerves, immune cells, and cancer cells within the TME that may underlie the resistance to current targeted therapies. Indeed, the discovery of tumor innervation, whereby tumors actively recruit nerves into the TME that contribute directly to tumorigenesis and tumor proliferation, has brought new perspectives to the study of tumor-nerve interactions. Whereas PNI refers to the passive invasion of neural tissue by tumor, tumor innervation implicates nerves as active participants in cancer progression. While the molecular mechanisms driving these processes remain largely unresolved, a growing body of evidence supports heterotypic cell-cell communication through small extracellular vesicles known as exosomes. In this Review, we highlight the current evidence for peripheral nerve-mediated cancer progression, and explore known and potential roles of exosomes in nerve-tumor signaling pathways within the TME as candidates for therapeutic targets.

Early evidence of nerve-mediated tumorigenesis. Early in vitro studies examining the effects of dorsal root ganglion (DRG) cells on cocultured prostate cancer cells demonstrated that nerve cells are capable of stimulating cancer cell proliferation (13). Inspired by the clinical association between beta blocker use and reduced cancer-specific mortality (14), Magnon and colleagues were the first to describe that chemical or mechanical ablation of sympathetic nerves leads to markedly decreased tumorigenesis in a mouse model of prostate cancer, and that $\beta$-adrenergic receptors are required for tumor development (15). In contrast, stimulation of the parasympathetic nervous system (PSNS) increased tumor metastasis and invasion, thereby enhancing late-stage cancer progression. Notably, the authors demonstrated that muscarinic receptor activity within the tumor stroma, rather than the tumor itself, was critical for the development of tumor invasion.

Studies in other cancer models have further strengthened the concept of divergent and tissue-specific roles of the PSNS and sympathetic nervous system (SNS) in cancer progression (16). As in prostate cancer, pancreatic tumors are highly innervated by both sympathetic and parasympathetic nerve fibers (17). Using a genetically engineered mouse model (GEMM) of pancreatic ductal adenocarcinoma (PDAC), Renz and colleagues showed that both mechanical sympathectomy and adrenergic receptor blockade significantly prolonged survival (18). Moreover, PDAC cells treated with norepinephrine expressed higher levels of nerve growth factor (NGF), leading to increased neurite outgrowth in a DRG coculture system. NGF was noted to contribute to both tumor innervation and tumor proliferation, independent of adrenergic signaling. Indeed, increased expression of NGF has been identified in a range of cancers and correlates with reduced survival (19-21).

In contrast to its prometastatic effects seen in prostate cancer, the PSNS appears to take on a protective role in PDAC, as subdiaphragmatic vagotomy enhances tumorigenesis (22). Moreover, treatment with bethanechol, a muscarinic agonist, limits the tumorigenic phenotype in vagotomized mice. The authors identified increased expression of the $\mathrm{M}_{1}$ muscarinic acetylcholine receptor (encoded by CHRM1) in response to vagotomy, and subsequently showed that $\mathrm{M}_{1}$ receptor activation suppresses MAPK and PI3K activity, thus contributing to tumor suppression (22). Taken together, these data illustrate that tumor behavior is directly impacted by both SNS and PSNS signals, which lead to neurotrophin release and subsequent recruitment of nerves in a feed-forward mechanism.
Beyond the autonomic nervous system, recent studies have explored contributions of sensory nerves to tumor growth in pancreatic, thyroid, head and neck, cervical, skin, and ovarian cancers (23-28). As in parasympathetic denervation of the pancreas, chemical denervation of sensory fibers by capsaicin in a PDAC GEMM leads to prolonged survival and decreased tumorigenesis while decreasing sensory nerve-mediated spinal inflammation (23). Similarly, sensory denervation by lingual nerve transection resulted in impaired tumor growth and neurogenesis in a murine model of oral cavity squamous cell carcinoma (26). The authors further demonstrated that Trp53 mutations in oral cancer cells induced a phenotypic switch in tumor-associated neurons from sensory to adrenergic, leading to increased tumor-nerve density and tumor growth. Furthermore, Vermeer and colleagues demonstrated that human head and neck squamous cell carcinomas harbor primarily sensory nerves, and that tumor innervation can be induced through exosomal delivery of the neurotrophin EphrinB1 (25). While the mechanism relating sensorial innervation and tumor potentiation is not fully known, substance $\mathrm{P}$ (SP), a nociceptive neurotransmitter, as well as its high-affinity receptor, NK-1R, have been shown to be highly expressed in both pancreatic and breast cancers and potentiate tumor growth $(29,30)$. Together, the data heretofore establish that peripheral nerves are present within a broad range of cancers and influence cancer behavior.

However, several important questions regarding neural contributions to cancer initiation and progression remain unresolved. For one, the provenance of neuronal tissue in tumors has been attributed to several sources: stimulation of preexisting locoregional nerves to produce new tumor-directed axons (axonogenesis); transdifferentiation of tumor cells to a neural phenotype (neurogenesis; refs. 31, 32); and recruitment of neural progenitor cells from the central nervous system (33). Reviewed in detail recently (16), the relative contributions of each lineage and its respective mechanisms warrant further investigation. Moreover, current evidence demonstrates that tumorinfiltrating nerves change in density and composition as tumor stage increases, and thus are likely to modulate tumor growth differently depending on the quantity and type of innervation (Figure 1). Since nerves are present throughout human tissues, they may participate in the earliest phases of tumor initiation and establishment of the TME. Additionally, nerve signaling at distant sites may be altered by tumor-derived signals to develop niches that promote metastatic deposition and growth (34). Thus, further study of tumor innervation will necessitate careful exploration of nerve-tumor interactions in both early and late development stages of cancers to identify clinically meaningful therapeutic targets.

\section{Neural contributions to the TME}

Neurotransmitters. Given their proximity, it stands to reason that tumor-associated nerves impact both cancer and noncancer cells within the TME to create a protumoral environment. Once a cancer cell has transformed and begun dividing, multiple metabolic and signaling changes must occur to support the clonal expansion, invasion, dissemination, and immune escape that confer lethality to a tumor. Thus, interactions between the nervous system and components of the TME are likely as critical as direct nerve-cancer cell communication in driving tumorigenesis. In that vein, a growing number of neurotrophic factors have been shown to be expressed by primary tumors and within the TME, leading to autocrine stimulation of 


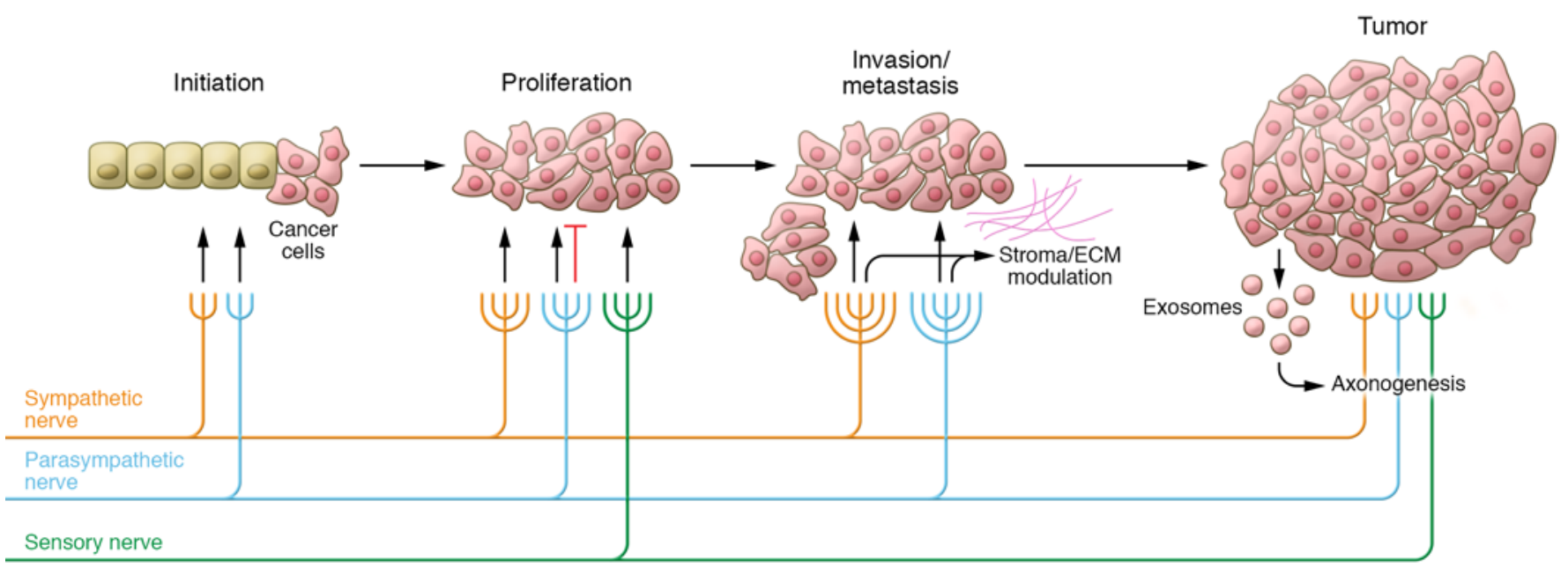

Figure 1. Tumor innervation. Local sympathetic, parasympathetic, and sensory nerves act on incipient cancer cells and the microenvironment to regulate proliferation and enhance metastasis. As tumors progress, new axons are recruited to the tumor microenvironment to further potentiate growth, via exosomes and other mechanisms. Tumor-nerve interactions are tissue specific; thus, interactions depicted are representative of observations in several cancer types.

tumor growth and an association with increased tumor-nerve density $(18,21)$. Grouped by their canonical functions, these molecules include neurotransmitters, neuropeptides, neurotrophins, and their respective receptors. While the responsible mechanisms appear to be tissue specific, neural signals within the TME lead to cellular changes that promote tumor growth, angiogenesis, immune modulation, and further recruitment of nerves to contribute to progression. A complete overview of neurotransmitters involved in cancer networks is provided elsewhere (35); neurotransmitters that have been directly implicated in tumor-nerve signaling are reviewed here.

The primary SNS catecholamine is norepinephrine, which binds to adrenergic receptors on numerous cell types to control critical functions such as DNA damage repair, apoptosis, and immune response. The clinically observed relationship between biobehavioral risk factors (depression, low social support) and increased intratumoral norepinephrine (36) led Allen and colleagues to define a role for norepinephrine-mediated tumorinnervationin ovariancancer (37). Using a daily restraint mouse model, they showed that stress led to increased tumor innervation and intratumoral norepinephrine levels. Importantly, intratumoral norepinephrine levels were not decreased by peripheral synaptic inhibition, suggesting that hematogenous norepinephrine delivery to the tumor is minimal compared with intratumoral sources. Moreover, norepinephrine exposure led to increased brainderived neurotrophic factor (BDNF) expression via $\beta$-adrenoreceptor 3 , which subsequently acts on its native receptor TrkB expressed on host nerve cells to drive tumor growth. Interestingly, inhibition of tumor TrkB signaling had no impact on restraint stress-mediated tumor growth. Findings were similar in murine breast cancer models, where tumor-specific sympathetic nerve stimulation led to increases in intratumoral norepinephrine and tumor growth that were reversed by sympathetic denervation or $\beta$-adrenergic antagonists (38). Moreover, in gastric cancer cells, $\beta$-adrenergic signaling increased expression of VEGF and MMP while upregulating STAT3 and ERK1/2JNK-MAPK signaling pathways, leading to increased metastasis in vivo (39). Therefore, nerves present within the tumor promote both further nerve recruitment and sustained adrenergic signaling in the TME, which together stimulate cancer progression.
Acetylcholine, the primary neurotransmitter of the PSNS, binds and activates either nicotinic acetylcholine receptors or muscarinic acetylcholine receptors (mAChRs, $\mathrm{M}_{1}-\mathrm{M}_{5}$, encoded by CHRM1-5), and has been similarly implicated in driving neurotransmittermediated tumor growth. Using a murine model of gastric cancer, Hayakawa and colleagues noted an increase in cholinergic nerve density associated with dysplastic epithelium, suggesting that acetylcholine is required for malignant transformation (40). They further showed that treating tumor cells with acetylcholine resulted in a significant increase in NGF production, and that forced expression of NGF in gastric mucosa led to increased nerve density and epithelial proliferation through activation of the NGF receptor TrkA (40). However, parasympathetic signaling through acetylcholine does not universally stimulate cancer progression. Genetic neurostimulation using a neuron-specific choline acetyltransferase resulted in decreased tumor growth of xenografted breast cancers, whereas pirenzepine, an $\mathrm{M}_{1}$ cholinergic antagonist, inhibited this effect (38). These contradictory observations may be reconciled by further investigation of the TME, as neurotransmitters and receptors present within other cell types may contribute substantially to nerve-mediated phenotypic effects. For example, the $\mathrm{M}_{1}$ receptor was absent in noncancer cells in the aforementioned breast cancer xenografts but highly expressed in tumor cells, suggesting that a direct nerve-tumor interaction is responsible for decreased proliferation in breast cancer (38). In contrast, the $\mathrm{M}_{1}$ receptor is expressed predominantly by stromal cells in prostate cancer, and when stimulated leads to basement membrane breakdown and increased tumor metastasis (15). Thus, the impacts of tumor innervation are highly dependent on both tumor type and the local environment, highlighting the need to evaluate components of the TME in addition to tumor cells in order to understand neural influences on cancer growth.

Innervation and angiogenesis. Angiogenesis, or the growth of new blood vessels into and around the tumor, is a hallmark of cancer and has been studied extensively (41). In human development, vasculature and peripheral nerves develop in parallel to establish circulation and neural communication throughout the body (42). Indeed, recent studies have shown that these processes are interdependent, 
and that crosstalk between axonal guidance molecules and endothelial cells can drive tumor angiogenesis (43). Thus, tumor nerves may exert angiogenic control within the TME. Recent work by Zahalka and colleagues has established a role for sympathetic signaling in prostate cancer angiogenesis (44). Using a GEMM of prostate cancer, the authors identified increasing levels of sympathetic tumor innervation and norepinephrine as tumors progressed from prostatic intraepithelial neoplasia to frank cancer, and found that these nerves became more closely associated with blood vessels. However, conditional deletion of ADRB2 in endothelial cells resulted in halted progression to cancer and reduced blood vessel density, branching, and migration. This occurred through increased expression of COA6, a cofactor in oxidative phosphorylation, leading to altered endothelial cell metabolism and decreased angiogenesis (44). Moreover, in a mouse model of ovarian cancer, chronic restraint stress leads to increased angiogenesis through activation of ADRB2 on tumor cells, leading to a rise in VEGF expression and concomitant angiogenesis and tumor growth (45). Similar findings of $\beta$-adrenergic receptor-mediated tumor growth have been reported in pancreatic (46) and lung cancer (47). Thus, norepinephrine can promote angiogenesis directly through endothelial cell activation, or indirectly by stimulating tumor secretion of VEGF.

Neuropeptides. While the roles of norepinephrine and acetylcholine are well defined in the autonomic nervous system, signals within the sensory and central nervous systems are more diverse, with a wide range of tissue-dependent effects. However, there is mounting evidence that neuropeptides also impact tumor innervation, growth, and angiogenesis. Neuropeptide calcitonin gene-related peptide (CGRP) has been shown to promote angiogenesis in both in vitro and in vivo models (48). In a CGRP ${ }^{-/-}$mouse model of lung cancer, tumor growth and angiogenesis were halted (48). Importantly, both competitive inhibition of CGRP and denervation of the tumor site in WT mice recapitulated the tumor-suppressive phenotype seen in the $\mathrm{CGRP}^{-/-}$mouse, thereby implicating sensory nerve-derived CGRP in tumor angiogenesis. Moreover, TRPA1, a sensory nerve ion channel, is highly expressed in prostate cancer stroma and when activated significantly upregulates VEGF secretion (49). SP is another neurotransmitter released by sensory nerves to promote local vasodilation and inflammation through mast cell activation (50). Its primary receptor, neurokinin-1 (NK-1R), is widely expressed throughout the body, and elevated expression within tumor cells and tumor-associated blood vessels has been documented $(51,52)$. Accordingly, NK-1 activation by SP induces angiogenesis both in vitro and in vivo through stimulation of endothelial cells $(53,54)$. In a murine model of pancreatic cancer, inhibition of SP signaling resulted in both decreased tumor volume and microvessel formation (55). Recently, work by Liu and colleagues examining corneal neovascularization demonstrated that sensory nerve-derived SP is upregulated in response to inflammatory stress and results in increased neovascularization in an NK-1R-dependent fashion, thus demonstrating direct neuronal control over angiogenesis (54). Since it is not robustly expressed by pancreatic cancer cells, SP within the TME is likely delivered by sensory nerves (52). Indeed, SP has been shown to be expressed in DRG neurite outgrowth, which stimulates pancreatic cancer cell proliferation and migration (52). Finally, neuropeptide Y (NPY), predominantly expressed in the central nervous system, has been implicated in the progression of several cancer types $(56,57)$, either directly by activation of specific receptors expressed on tumor cells or indirectly by enhancing angiogenesis (58). Most recently in prostate cancer, levels of NPY-positive nerves were increased in human preneoplastic tissues, and this was found to be an independent predictor of poor outcome (59). Moreover, prostate cancer cells were shown to increase NPYspecific neurite outgrowth, while inhibition of NPY signaling resulted in decreased cancer cell migration, increased apoptosis, and altered energy metabolism (59). Taken together, these data highlight the potential of sensory nerves and neuropeptides to enhance tumor growth directly, while facilitating axonogenesis to further potentiate nerve-mediated signaling in the TME.

Neuroimmune regulation in the TME. With respect to cancer, the immune system represents a double-edged sword. Whereas $\mathrm{CD}^{+} \mathrm{T}$ cells and NK cells serve to detect and kill both foreign cells and cells harboring aberrant expression of cell surface proteins, numerous cytokines and inflammatory signals can induce antiapoptotic responses and drive proliferation in cancer cells. T cells express cell surface receptors, including PD- 1 and CTLA-4, that inhibit the immune response when bound to ligand on cells in the physiologic state. Likewise, cancer cells that express high levels of the PD-1 ligand PD-L1 escape immune surveillance through PD-1/ PD-L1-induced inhibition of PI3K/AKT signaling and suppressed $\mathrm{T}$ cell activation (60). TME infiltration by immune cells is clinically relevant, as the presence of intratumoral $\mathrm{CD}^{+}$and $\mathrm{CD}^{+} \mathrm{T}$ cells correlates with decreased risk of colorectal cancer recurrence (61), increased survival in ovarian cancer (62), and positive response to immune checkpoint inhibitors (63-66). Aside from T cells, the TME also contains Tregs, macrophages, myeloid-derived suppressor cells (MDSCs), and dendritic cells that contribute to the inflammatory milieu that either drives or suppresses tumor growth (67).

Furthermore, immune cells express receptors for a wide range of neurotransmitters and neuropeptides, which under physiologic conditions facilitate cell activation by nerve-derived neurotransmitters in primary and secondary lymphoid organs $(68,69)$. Thus, tumor infiltrating nerves may exert similar control over immune cells within the TME. Tumor-infiltrating lymphocytes (TILs) are considered to be the primary actors in tumor immune surveillance and responsible for the antitumor activity of immune checkpoint inhibitors across a range of cancers (70). Kamiya and colleagues investigated the influence of tumoral autonomic nerve signaling on immune checkpoint receptor expression and found that either tumor-specific sympathetic denervation or parasympathetic stimulation results in decreased expression of PD-1 and FOXP3 in TILs (38). This effect was observed in both transplanted and induced or spontaneous mouse models of breast cancer, and resulted in increased tumor growth and metastasis (38). In prostate cancer, tumor-associated nerves have been shown to express PD-L1, with increased density of PD-L1-positive nerves correlating with worse prognosis (71). While the mechanism driving altered immune checkpoint expression is as yet unresolved, these data provide strong support for neural control over tumor immunity within the TME. Furthermore, in another mouse model of breast cancer, both stress-induced and pharmacologic $\beta$-adrenergic activation induced macrophage infiltration into tumor parenchyma and differentiation to the immunosuppressive $\mathrm{M}_{2}$ phenotype (72). This resulted in increased expression of TGF- $\beta$, VEGF, and MMP- 9 that enhanced angiogenesis and metastasis (72). In the same system, small molecule inhibition of macrophage recruitment reversed the stress-induced 


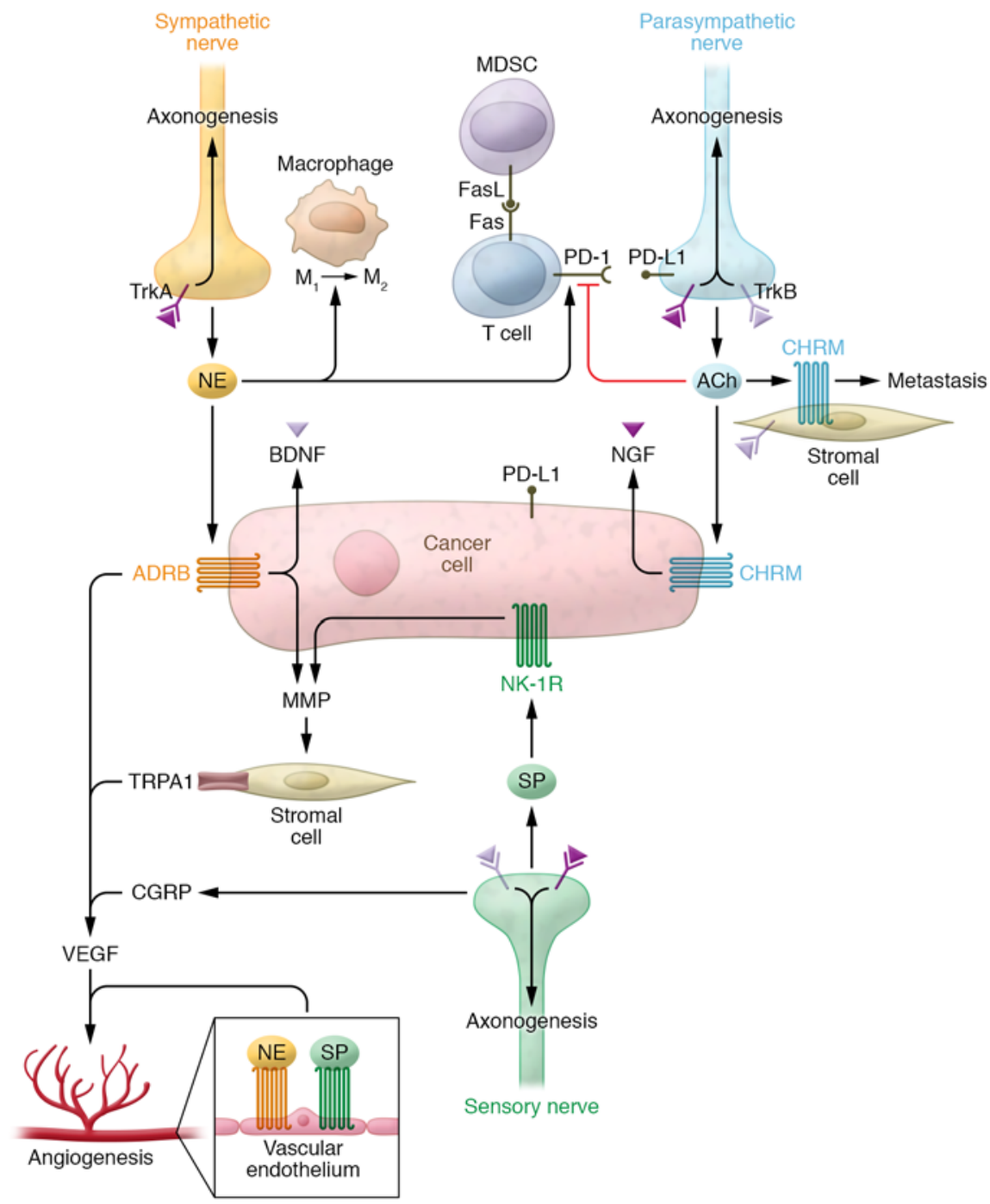

Figure 2. Neural communication in the TME. Neurotrophins (NGF, BDNF), neurotransmitters (norepinephrine [NE], acetylcholine [ACh]), neuropeptides (SP, CCRP), and their cognate receptors modulate activity of cancer cells and components of the TME. Norepinephrine induces cancer cell proliferation and production of neurotrophins, while also inducing angiogenesis in endothelial cells and alternative $\left(M_{2}\right)$ activation of macrophages. Neurotrophins further induce axonogenesis through activation of TrkA/B expressed on nerve cells. T cell activity and expression of cell death receptors (FAS/PD-1) on $T$ cells and cancer cells (PD-L1) are modulated by neurotransmitters, altering tumor immunity within the TME. Stromal cells also respond to neuropeptides to alter ECM composition via expression of MMPs, which regulate invasion and metastasis.

increases in macrophage infiltration, angiogenesis, and metastasis. Thus, adrenergic signaling in the TME may indirectly promote cancer progression through modulation of macrophage activity. The presence of suppressive macrophages also impacts TIL function within the TME. Elegant work by Zhu and colleagues using both induced and transplanted mouse models of melanoma demonstrated that induced tumors recruited high levels of polymorphonuclear MDSCs, while transplanted tumors did not (73). This subpopulation of MDSCs expressed increased levels of FasL, which binds the Fas receptor on TILs, leading to apoptosis. Cotreatment using anti-PD-1 and antiCTLA-4 immune checkpoint inhibition and FasL-neutralizing antibody resulted in decreased tumor growth compared with immune checkpoint inhibition alone, suggesting that tumor killing by TILs is limited by recruited MDSCs. Taken together, these results indicate that nerves and neurotransmitters in the TME influence immune cell recruitment and function within a tumor to impact cancer progression (Figure 2).

Moreover, these studies highlight the difficulties in reproducing the complete TME using transplanted tumor models, as autochthonous tumors and transplanted isogenic tumors develop distinct TMEs with clinically relevant differences, likely due to critical signaling networks that shape the TME from the earliest stages of tumorigenesis. It is equally likely that tumor-nerve interactions influence early cancer development, and thus autochthonous models will serve as important tools in understanding neural influences on the TME and cancer.

\section{Exosomes as novel regulators of the TME}

The data presented thus far illustrate the central concepts of tumor innervation: neural input alters the initiation, growth, and metastatic potential of cancers; tumors are capable of recruiting nerves from surrounding tissue into the TME; and neural signaling modulates the TME to alter tumor immunity, angiogenesis, the ECM, and stroma to promote cancer growth. However, several questions remain unresolved, including the precise signaling mechanisms by which nerves induce tumorigenesis, the role nerves play in controlling the TME, and how nerves and tumor innervation affect the development of metastases that ultimately confer lethality to a tumor.

While further study is required, preliminary evidence supports a role for exosomes in several processes of tumor innervation. Exosomes are small, membrane-bound vesicles $(30-150 \mathrm{~nm})$ released by all cells. Exosome content is dependent on the cell of origin, and while the mechanisms of exosome biogenesis are still being characterized, it is a nonrandom process involving several energy-dependent steps that preferentially package proteins, lipids, DNAs, and RNAs for secretion (74). Once released by a cell, exosomes are capable of traveling both short (within the TME) and long distances (hematogenous dissemination) to reach target cells. Exosomes are then taken up where the cargo can exert biological effects, including promotion of metastasis and resistance to chemotherapy (75-78). The exosome, then, is well-positioned to be a mediator of local oncogenic processes by potentiating cell-cell communication, while also traveling to distant sites to establish metastatic niches that can be seeded by malignant cells (79). Indeed, numerous studies have shown increased exosome concentration and altered cargo in patients with cancer, raising the potential for exosomes to serve as cancer biomarkers $(80,81)$. 


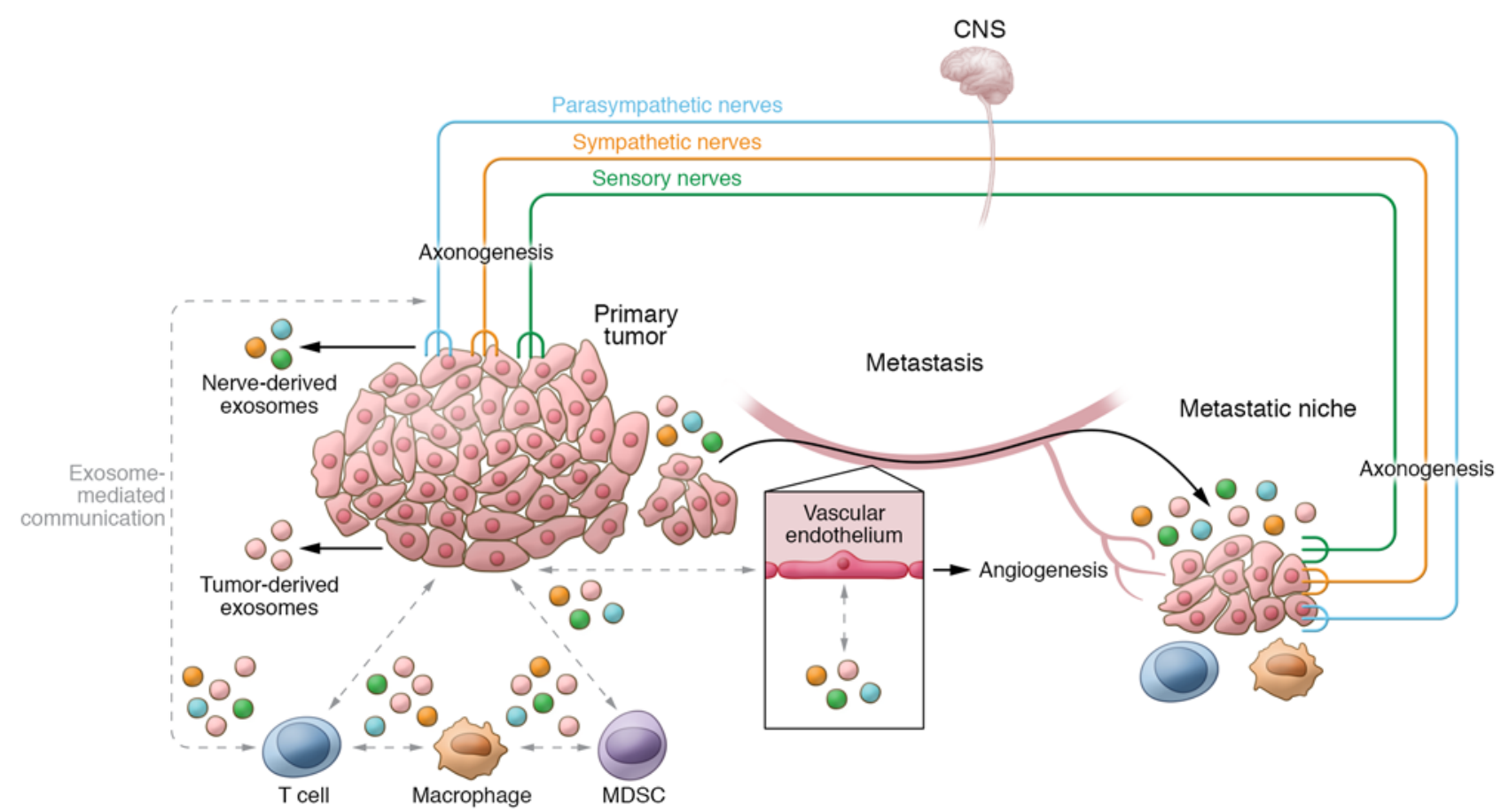

Figure 3. Exosomes as intercellular messengers between nerves and the TME. Exosomes are capable of local and distant signaling. They can induce axonogenesis of nerve fibers into the TME and modulate tumor growth. Tumor-derived exosomes (TEX) also influence T cell function within the TME, leading to an immune-suppressive environment. Nerves are also capable of both exosome release and internalization, and thus may also participate in exosome-mediated communication within the TME. TEX have been implicated in developing the metastatic niche; however, it is not yet known whether altered neural signaling, either locally or via the central nervous system, is in part responsible for promoting the growth of seeded cells into metastatic tumors. Identification of exosome-mediated signaling may lead to novel treatments to limit angiogenesis, tumor immunity, metastasis, and cancer pain.

Moreover, exosomes remain stable in serum and are preferentially released and taken up under the low $\mathrm{pH}$ conditions typical of the TME (82). Accordingly, exosomes have been increasingly identified as important actors in cancer biology, including having direct roles in modulating tumor immune response $(83,84)$ and angiogenesis $(85)$.

Exosomes in nerve physiology and tumor innervation. Exosomes are released by nervous system cells under normal and pathologic conditions, facilitating cell-cell communication during development as well as in tissue repair and modulation of synaptic activity (86). In the peripheral nervous system, Schwann cells serve to support and myelinate nerves while regulating axon growth and regeneration through complex nerve-Schwann cell interactions. This occurs through Schwann cell release of exosomes containing p75 neurotrophin receptor, which are taken up by DRG cells, leading to enhanced axon regeneration both in vitro and in vivo (87). Schwann cellderived exosomes are known to be internalized by peripheral axons (87), where they may act on microRNAs (miRs) or mRNAs governing axon growth that are preferentially stored in axon terminals (88). In response to injury, repair Schwann cells express c-Jun and Sox2. This leads to upregulation of exosomal miR-21, a now well-established oncomiR that inhibits the tumor suppressor PTEN (87) and is upregulated in many cancers (89). Another study, by Ching and colleagues, showed that both Schwann cell and Schwann cell-like differentiated adipose stem cells produce exosomes containing numerous miRs and mRNAs that control axon growth (90). Moreover, exosomes derived from cerebrospinal fluid containing miRs induce mTOR signaling in recipient cells, leading to neuronal proliferation (91). Thus, it is clear that exosomes fulfill several physiologic roles in the nervous system, giving rise to the premise that tumor-nerve crosstalk via exosomes can activate nerve development and regeneration pathways to enhance tumor growth.

Since exosomes mediate axonal guidance independent of cell contact (92), tumors may recruit nerves into the TME through exosomal delivery of neurotrophic factors. As noted earlier, production of NGF and BDNF is well documented in several cancer types (18, 37, 93), and correlates clinically with densely innervated tumors and poor outcomes. Conversely, NGF blockade results in significant attenuation of tumor growth and metastasis (19). Thus, neurotrophic factors not only recruit nerves to the TME, but also directly impact tumor cells to promote tumor growth (94). Despite the inherent difficulties of isolating the biological effects of exosomes within the TME, several lines of evidence suggest they play an important role in neurotrophic signal conduction. Circulating exosomes from patients with head and neck cancers promote neurite outgrowth in vitro when compared with those from healthy control individuals (25). Moreover, in a murine model of human papillomavirus-induced head and neck cancer, genomic knockdown of exosome release significantly decreased tumor growth and tumor innervation (25). When sensory nerves were experimentally ablated in the same system, tumor growth was likewise attenuated. Interestingly, the axonogenic potential of tumor-derived exosomes (TEX) was not impacted by NGF neutralization, and NGF was not detected in TEX despite robust expression by tumor cells. However, EphrinB1, an axonal guidance molecule,wasidentifiedinTEX, andexosomesreleasedfromEphrinB1overexpressing cells induced significantly higher neurite outgrowth compared with parental lines. Thus, TEX may induce tumor 
innervation through multiple pathways involved in neurodevelopment. A recent study examining TEX from oral cavity squamous cell carcinoma cells confirmed the ability of TEX to induce neurite outgrowth (26). The authors further showed that p53-deficient cells release miR-34a-3p-deficient TEX, leading to increased intratumoral norepinephrine and axonogenesis of cocultured DRG neurons (26). While a specific target of miR-34a-3p was not identified, analysis of differentially expressed genes revealed significant enrichment of transcription factors associated with neuron outgrowth, differentiation, and axon guidance, suggesting that TEX-delivered miRs, or lack thereof, influence neuronal differentiation and activity (26).

TEX may also induce innervation indirectly through interaction with other components of the TME. For example, fibroblasts cocultured with salivary gland cancer-derived exosomes express higher levels of NGF, BDNF, and the BDNF receptor NTRK2, leading to increased tumor invasiveness in an NGF-dependent manner (95). While these studies were conducted in vitro in the absence of a TME, the ability of TEX to directly upregulate neurotrophin, proinflammatory cytokine, and chemokine secretion suggests that exosomemediated signaling has the potential to induce tumor innervation and nerve-mediated protumoral effects. Further investigation in vivo, ideally using inducible or spontaneous cancer models with a complete TME, is required in order to clarify the effects of TEX in the TME.

Can exosomes mediate nerve-immune cell regulation? Once established in the TME, nerves are free to interact with other cells present in the environment and modulate their activity, including immune cells. Despite several putative mechanisms, the role of exosomes in this capacity has yet to be elucidated, and further study is warranted. In the central nervous system, numerous lines of evidence support exosome-mediated communication between microglia, oligodendrocytes, and neurons through delivery of miRs, inflammatory cytokines, and other proteins that contribute to homeostasis and regulation of neurotransmitter release (96). While there are no published data documenting peripheral nerve-immune cell communication via exosomes in the context of cancer, head and neck TEX are capable of inducing an immune suppressor phenotype in human $\mathrm{CD}^{+} \mathrm{T}$ cells, illustrating that critical $\mathrm{T}$ cell functions in cancer can be manipulated by exosomes (97). Additionally, melanomaderived exosomes containing PD- 1 are $\mathrm{CD}^{+} \mathrm{T}$ cell suppressive and correlate with poor response to immune checkpoint inhibition (98). Given that nerves can express either PD-1 (99) or PD-L1 (71), tumor immunity may be modulated by exosome-mediated delivery within the TME. Further evidence of nerve-immune cell crosstalk can be gleaned from the field of neuroregeneration, where nerves and Schwann cells secrete inflammatory factors to recruit macrophages in response to injury. Indeed, Simeoli and colleagues demonstrated that the level of miR-21 is significantly increased in DRGs in response to sciatic nerve injury (100). They further showed that DRG stimulation with capsaicin leads to increased miR-21 expression in exosomes, which subsequently induce a proinflammatory phenotype switch in recipient macrophages. Moreover, Sprouty2, a target of miR-21, was also downregulated in macrophages, clearly demonstrating that neuron-derived, exosomal miRs are functional in recipient cells (100). Thus, the components required for nerveimmune cell crosstalk via exosomes have been established, and efforts should be made to delineate the importance of neural control on immune function within the TME.

\section{Concluding remarks}

Tumor innervation and neural control of the TME have the potential to explain several key observations that have perplexed cancer researchers for decades. In particular, the presence and activity of nerves within tumors may underlie the increased incidence and mortality of cancer in patients with high psychosocial stress (101). Critically, the addition of nerves to the TME landscape provides an alternative system, capable of signaling both within and outside the TME, whereby cancers can overcome otherwise logically designed and specific drugs that should stop cancer in its tracks. For example, if a tumor is exposed to antiangiogenic treatment, nerves within the TME may respond to hypoxic stimuli and induce production of neurotransmitters or neurotrophins that restore angiogenic properties to the tumor. Indeed, it is interesting to note that the highly innervated tumors discussed are generally insensitive to anti-VEGF therapy, and significant overall survival benefit has not been seen across several trials, particularly in prostate, pancreatic, and ovarian cancers $(3,4,102)$.

The data presented herein provide a rationale for targeting the nervous system as a means to control cancer growth. However, important features of tumor innervation need further characterization prior to rational clinical trial design. For one, priority should be placed on determining the chronology of tumor innervation and the relative impact of tumor nerves at each stage of cancer development. As neural inputs are cancer specific, a more complete understanding of tumor-nerve content and function across cancer types is required in order to identify the optimal timing and mode of intervention. Targeting of tumor innervation can be broadly categorized into three strategies. Denervation, either by surgical transection or chemical ablation, is an obvious but limited approach for solid tumors that display nerve-mediated growth. While preclinical evidence supports the efficacy of this approach (15), anatomical and technical limitations and the potential for off-target effects will likely limit its applicability to select disease sites. Moreover, the timing of denervation influences cancer development (15) and thus may be less effective in patients presenting with advanced disease. Nevertheless, phase I clinical trials of chemical denervation are underway, with promising results (103). A more broadly applicable strategy to limit tumor innervation is targeting of tumor-mediated axonogenic signaling. Recent developments in precision therapy have produced FDA-approved specific inhibitors of NGF (anti-NGF mAb; tanezumab), and Trk receptors (pan-TRK small molecule inhibitor; entrectinib, larotrectinib) (104-107). While these drugs were designed to target pro-oncogenic tumor expression of Trk proteins, they may also impact tumor nerve recruitment by interfering with the NGF/Trk signaling axis. For cancers that demonstrate increased innervation in recurrent or posttreatment settings, antineurotrophic therapies present an attractive option for mitigating chemoresistance and prolonging remission. Finally, modulation of nerve-mediated signaling within the TME holds the potential for broad applicability across all nerve-bearing tumors. While further delineation of specific signaling mechanisms is required, the US pharmacopeia is replete with neuromodulatory drugs that could be repurposed to provide synergy with conventional treatments. As mentioned above, beta blockers are associated with prolonged survival in several cancers $(108,109)$ and modulate cancer progression in 
animal models, results that together provide a sound rationale for adjuvant beta blockade. Similarly, drugs that specifically target SP (aprepitant, NK-1 antagonist), GABA (gabapentinoids), and CGRP (eptinezumab, erenumab) may be repurposed as adjuvant therapies to modulate the neural component of the TME.

While exosomes are still being explored in the context of tumor innervation and neural control of the TME, they hold both diagnostic and therapeutic potential in the treatment of cancer. Advances in bioinformatics and exosome isolation technologies are already enhancing the performance of exosome-based liquid biopsies $(81,110)$. On the other hand, preclinical evidence suggests that exosomes bear the potential to become custom-designed drug delivery systems, in which exosomes are loaded with cytotoxic or immunomodulatory factors and targeted toward tumor cells $(111,112)$. Thus, as our understanding of the intersection between tumors, nerves, and the immune system evolves, intercellular communication via exosomes will be an attractive target for anticancer therapies to limit nerve-mediated tumor growth, angiogenesis, metastasis, and immune evasion (Figure 3).

Altogether, tumor innervation and neural activity within the TME are emerging concepts in cancer biology that tie together most, if not all, major systems that govern cancer behavior. While it is clear that nerves impact multiple cancer functions, further research across cancer types is needed in order to understand the magnitude and mechanism of tumor-nerve-TME crosstalk and the natural history of tumor innervation. Once a more complete landscape is elucidated, myriad platforms for therapeutic intervention exist that can be rapidly tested in an adjuvant fashion, providing hope for prolonging survival of patients with cancer, improving cancer-related pain management, and transitioning away from crude and ineffective cytotoxic therapies.

\section{Acknowledgments}

The authors would like to thank Hunter D. Reavis and Valerie L. Luks for their suggestions and critical reading of the manuscript and Michael Cooper (Cooper Graphics \& Fine Art; http://www. cooper247.com/) for assistance with the illustrations. This work was supported by NIH SPORE P50 CA228991 (RD), the US Department of Defense (OC170094, OC180420), the Dr. Miriam and Sheldon G. Adelson Medical Research Foundation (RD), the Honorable Tina Brozman Foundation for Ovarian Cancer Research (RD), The V Foundation (RD), the Basser Center for BRCA (RD), the Claneil Foundation (RD), and the Skacel Family Scientific Scholar Award from the Rivkin Center (SMG).

Address correspondence to: Ronny Drapkin, Department of Obstetrics and Gynecology, University of Pennsylvania Perelman School of Medicine, Biomedical Research Building II/III, Suite 1215, 421 Curie Boulevard, Philadelphia, Pennsylvania 19104, USA. Phone: 215.746.3973; Email: rdrapkin@pennmedicine.upenn.edu.
1. Hanahan D, Weinberg RA. The hallmarks of cancer. Cell. 2000;100(1):57-70.

2. Hanahan D, Weinberg RA. Hallmarks of cancer: the next generation. Cell. 2011;144(5):646-674.

3. Tewari KS, et al. Final overall survival of a randomized trial of bevacizumab for primary treatment of ovarian cancer. J Clin Oncol. 2019;37(26):2317-2328.

4. Van Cutsem E, et al. Phase III trial of bevacizumab in combination with gemcitabine and erlotinib in patients with metastatic pancreatic cancer. J Clin Oncol. 2009;27(13):2231-2237.

5. Schachter J, et al. Pembrolizumab versus ipilimumab for advanced melanoma: final overall survival results of a multicentre, randomised, open-label phase 3 study (KEYNOTE-006). Lancet. 2017;390(10105):1853-1862.

6. Govindan R, et al. Phase III trial of ipilimumab combined with paclitaxel and carboplatin in advanced squamous non-small-cell lung cancer. JClin Oncol. 2017;35(30):3449-3457.

7. Wolchok JD, et al. Updated results from a phase III trial of nivolumab (NIVO) combined with ipilimumab (IPI) in treatment-naive patients (pts) with advanced melanoma (MEL) (CheckMate 067). J Clin Oncol. 2016;34(15_suppl):9505.

8. De Sousa Pereira A. A basis for sympathectomy for cancer of the cervix uteri. Arch Surg. 1946;52(3):260-285.

9. Ernst P. Uber das Wachstum und die Verbreitung bosartiger Gechwulste insbesondere des Krebs in den Lymphbahnen der Nerven. Beitr Path Anat. 1905; Suppl 7:29-51.

10. Chen SH, et al. Perineural invasion of cancer: a complex crosstalk between cells and molecules in the perineural niche. Am J Cancer Res.
2019;9(1):1-21.

11. van Wyk HC, et al. The role of perineural invasion in predicting survival in patients with primary operable colorectal cancer: a systematic review. Crit Rev Oncol Hematol. 2017;112:11-20.

12. Zhu J, et al. Perineural invasion as a prognostic factor in head and neck squamous cell carcinoma: a systematic review and meta-analysis. Acta Otolaryngol. 2019;139(11):1038-1043.

13. Ayala GE, et al. In vitro dorsal root ganglia and human prostate cell line interaction: redefining perineural invasion in prostate cancer. Prostate. 2001;49(3):213-223.

14. Grytli HH, et al. Use of $\beta$-blockers is associated with prostate cancer-specific survival in prostate cancer patients on androgen deprivation therapy. Prostate. 2013;73(3):250-260.

15. Magnon C, et al. Autonomic nerve development contributes to prostate cancer progression. Science. 2013;341(6142):1236361.

16. Reavis HD, et al. Tumor innervation: cancer has some nerve. Trends Cancer. 2020;6(12):1059-1067.

17. Borden $\mathrm{P}$, et al. Sympathetic innervation during development is necessary for pancreatic islet architecture and functional maturation. Cell Rep. 2013;4(2):287-301.

18. Renz BW, et al. beta2 adrenergic-neurotrophin feedforward loop promotes pancreatic cancer. Cancer Cell. 2018;33(1):75-90.

19. Adriaenssens E, et al. Nerve growth factor is a potential therapeutic target in breast cancer. Cancer Res. 2008;68(2):346-351.

20. Ye Y, et al. Nerve growth factor links oral cancer progression, pain, and cachexia. Mol Cancer Ther. 2011;10(9):1667-1676.

21. Pundavela J, et al. ProNGF correlates with
Gleason score and is a potential driver of nerve infiltration in prostate cancer. Am J Pathol. 2014;184(12):3156-3162.

22. Renz BW, et al. Cholinergic Signaling via muscarinic receptors directly and indirectly suppresses pancreatic tumorigenesis and cancer stemness. Cancer Discov. 2018;8(11):1458-1473.

23. Saloman JL, et al. Ablation of sensory neurons in a genetic model of pancreatic ductal adenocarcinoma slows initiation and progression of cancer. Proc Natl Acad Sci U S A . 2016;113(11):3078-3083.

24. Lucido CT, et al. Innervation of cervical carcinoma is mediated by cancer-derived exosomes. Gynecol Oncol. 2019;154(1):228-235.

25. Madeo M, et al. Cancer exosomes induce tumor innervation. Nat Commun. 2018;9(1):4284.

26. Amit M, et al. Loss of p53 drives neuron reprogramming in head and neck cancer. Nature. 2020;578(7795):449-454.

27. Peterson SC, et al. Basal cell carcinoma preferentially arises from stem cells within hair follicle and mechanosensory niches. Cell Stem Cell. 2015;16(4):400-412.

28. Rowe CW, et al. Innervation of papillary thyroid cancer and its association with extra-thyroidal invasion. Sci Rep. 2020;10(1):1539.

29. Garcia-Recio S, et al. Substance P autocrine signaling contributes to persistent HER2 activation that drives malignant progression and drug resistance in breast cancer. Cancer Res. 2013;73(21):6424-6434.

30. Sinha S, et al. PanIN neuroendocrine cells promote tumorigenesis via neuronal cross-talk. Cancer Res. 2017;77(8):1868-1879.

31. Farach A, et al. Neuronal trans-differentiation in prostate cancer cells. Prostate. 2016;76(14):1312-1325. 
32. Lapuk AV, et al. From sequence to molecular pathology, and a mechanism driving the neuroendocrine phenotype in prostate cancer. J Pathol. 2012;227(3):286-297.

33. Mauffrey P, et al. Progenitors from the central nervous system drive neurogenesis in cancer. Nature. 2019;569(7758):672-378.

34. Ayala GE, et al. Cancer-related axonogenesis and neurogenesis in prostate cancer. Clin Cancer Res. 2008;14(23):7593-7603.

35. Jiang SH, et al. Neurotransmitters: emerging targets in cancer. Oncogene. 2020;39(3):503-515.

36. Lutgendorf SK, et al. Depression, social support, and beta-adrenergic transcription control in human ovarian cancer. Brain Behav Immun. 2009;23(2):176-183.

37. Allen JK, et al. Sustained adrenergic signaling promotes intratumoral innervation through BDNF induction. Cancer Res. 2018;78(12):3233-3242.

38. Kamiya A, et al. Genetic manipulation of autonomic nerve fiber innervation and activity and its effect on breast cancer progression. Nat Neurosci. 2019;22(8):1289-1305

39. Zhang X, et al. Chronic stress promotes gastric cancer progression and metastasis: an essential role for ADRB2. Cell Death Dis. 2019;10(11):788.

40. Hayakawa Y, et al. Nerve growth factor promotes gastric tumorigenesis through aberrant cholinergic signaling. Cancer Cell. 2017;31(1):21-34.

41. De Palma M, et al. Microenvironmental regulation of tumour angiogenesis. Nat Rev Cancer. 2017;17(8):457-474.

42. Carmeliet P, Tessier-Lavigne M. Common mechanisms of nerve and blood vessel wiring. Nature. 2005;436(7048):193-200.

43. Klagsbrun M, Eichmann A. A role for axon guidance receptors and ligands in blood vessel development and tumor angiogenesis. Cytokine Growth Factor Rev. 2005;16(4-5):535-548.

44. Zahalka AH, et al. Adrenergic nerves activate an angio-metabolic switch in prostate cancer. Science. 2017;358(6361):321-326.

45. Thaker PH, et al. Chronic stress promotes tumor growth and angiogenesis in a mouse model of ovarian carcinoma. Nat Med.2006;12(8):939-944.

46. Kim-Fuchs C, et al. Chronic stress accelerates pancreatic cancer growth and invasion: a critical role for beta-adrenergic signaling in the pancreatic microenvironment. Brain Behav Immun. 2014;40:40-47.

47. Melamed R, et al. Marginating pulmonary-NK activity and resistance to experimental tumor metastasis: suppression by surgery and the prophylactic use of a beta-adrenergic antagonist and a prostaglandin synthesis inhibitor. Brain Behav Immun. 2005;19(2):114-126.

48. Toda M, et al. Neuronal system-dependent facilitation of tumor angiogenesis and tumor growth by calcitonin gene-related peptide. Proc Natl Acad Sci U S A. 2008;105(36):13550-13555.

49. Derouiche $S$, et al. Activation of TRPA1 channel by antibacterial agent triclosan induces VEGF secretion in human prostate cancer stromal cells. Cancer Prev Res (Phila). 2017;10(3):177-187.

50. Suzuki R, et al. Direct neurite-mast cell communication in vitro occurs via the neuropeptide substance P. JImmunol. 1999;163(5):2410-2415.

51. Hennig IM, et al. Substance-P receptors in human primary neoplasms: tumoral and vascular localization. Int J Cancer. 1995;61(6):786-792.

52. Li X, et al. Neurotransmitter substance $P$ mediates pancreatic cancer perineural invasion via NK-1R in cancer cells. Mol Cancer Res. 2013;11(3):294-302.

53. Ziche M, et al. Substance P stimulates neovascularization in vivo and proliferation of cultured endothelial cells. Microvasc Res. 1990;40(2):264-278.

54 . Liu L, et al. Sensory neurons directly promote angiogenesis in response to inflammation via substance $\mathrm{P}$ signaling. FASEB J. 2020;34(5):6229-6243.

55. Guha $S$, et al. Broad-spectrum $G$ protein-coupled receptor antagonist, [D-Arg1,D-Trp5,7,9,Leu11]SP: a dual inhibitor of growth and angiogenesis in pancreatic cancer. Cancer Res. 2005;65(7):2738-2745.

56. Ruscica $\mathrm{M}$, et al. Role of neuropeptide $\mathrm{Y}$ and its receptors in the progression of endocrine-related cancer. Peptides. 2007;28(2):426-434.

57. Ruscica M, et al. Activation of the Y1 receptor by neuropeptide $Y$ regulates the growth of prostate cancer cells. Endocrinology. 2006;147(3):1466-1473.

58. Alasvand M, et al. Effect of blocking of neuropeptide Y Y2 receptor on tumor angiogenesis and progression in normal and diet-induced obese C57BL/6 mice. Glob J Health Sci. 2015;7(7 Spec No):69-78.

59. Ding Y, et al. Neuropeptide Y nerve paracrine regulation of prostate cancer oncogenesis and therapy resistance. Prostate. 2021;81(1):58-71.

60. Riley JL. PD-1 signaling in primary T cells. Immunol Rev. 2009;229(1):114-125.

61. Pages F, et al. International validation of the consensus Immunoscore for the classification of colon cancer: a prognostic and accuracy study. Lancet. 2018;391(10135):2128-2139.

62. Zhang L, et al. Intratumoral T cells, recurrence, and survival in epithelial ovarian cancer. $N$ EnglJ Med. 2003;348(3):203-213.

63. Fu Q, et al. Prognostic value of tumor-infiltrating lymphocytes in melanoma: a systematic review and meta-analysis. Oncoimmunology. 2019;8(7):1593806.

64. Yao W, et al. The prognostic value of tumor-infiltrating lymphocytes in hepatocellular carcinoma: a systematic review and meta-analysis. Sci Rep. 2017;7(1):7525.

65. de Ruiter EJ, et al. The prognostic role of tumor infiltrating T-lymphocytes in squamous cell carcinoma of the head and neck: a systematic review and meta-analysis. Oncoimmunology. 2017;6(11):e1356148.

66. Hodi FS, et al. Improved survival with ipilimumab in patients with metastatic melanoma. $\mathrm{NEnglJ}$ Med. 2010;363(8):711-723.

67. Gajewski TF, et al. Innate and adaptive immune cells in the tumor microenvironment. Nat Immunol. 2013;14(10):1014-1022.

68. Dantzer R. Neuroimmune interactions: from the brain to the immune system and vice versa. Physiol Rev. 2018;98(1):477-504.

69. Kuol N, et al. Crosstalk between cancer and the neuro-immune system. J Neuroimmunol. 2018;315:15-23.

70. Chen L, Han X. Anti-PD-1/PD-L1 therapy of human cancer: past, present, and future. JClin Invest. 2015;125(9):3384-3391.

71. Mo RJ, et al. Expression of PD-L1 in tumor-as- sociated nerves correlates with reduced $\mathrm{CD} 8^{+}$ tumor-associated lymphocytes and poor prognosis in prostate cancer. Int J Cancer. 2019;144(12):3099-3110.

72. Sloan EK, et al. The sympathetic nervous system induces a metastatic switch in primary breast cancer. Cancer Res. 2010;70(18):7042-7052.

73. Zhu J, et al. Resistance to cancer immunotherapy mediated by apoptosis of tumor-infiltrating lymphocytes. Nat Commun. 2017;8(1):1404.

74. Becker A, et al. Extracellular vesicles in cancer: cell-to-cell mediators of metastasis. Cancer Cell. 2016;30(6):836-848.

75. Valadi H, et al. Exosome-mediated transfer of mRNAs and microRNAs is a novel mechanism of genetic exchange between cells. Nat Cell Biol. 2007;9(6):654-659.

76. Zomer A, et al. In Vivo imaging reveals extracellular vesicle-mediated phenocopying of metastatic behavior. Cell. 2015;161(5):1046-1057.

77. Fabbri M, et al. MicroRNAs bind to Toll-like receptors to induce prometastatic inflammatory response. Proc Natl Acad Sci U S A. 2012;109(31):E2110-E2116.

78. Crow J, et al. Exosomes as mediators of platinum resistance in ovarian cancer. Oncotarget. 2017;8(7):11917-11936

79. Hoshino A, et al. Tumour exosome integrins determine organotropic metastasis. Nature. 2015;527(7578):329-335.

80. Taylor DD, Gercel-Taylor C. MicroRNA signatures of tumor-derived exosomes as diagnostic biomarkers of ovarian cancer. Gynecol Oncol. 2008;110(1):13-21.

81. Zhang P, et al. Ultrasensitive detection of circulating exosomes with a 3D-nanopatterned microfluidic chip. Nat Biomed Eng. 2019;3(6):438-451.

82. Parolini I, et al. Microenvironmental $\mathrm{pH}$ is a key factor for exosome traffic in tumor cells. J Biol Chem. 2009;284(49):34211-34222.

83. Muller L, et al. Tumor-derived exosomes regulate expression of immune function-related genes in human T cell subsets. Sci Rep. 2016;6:20254.

84. Greening DW, et al. Exosomes and their roles in immune regulation and cancer. Semin Cell Dev Biol. 2015;40:72-81.

85. Ribeiro MF, et al. Exosomes function in pro- and anti-angiogenesis. Curr Angiogenes. 2013;2(1):54-59.

86. Zappulli V, et al. Extracellular vesicles and intercellular communication within the nervous system. J Clin Invest. 2016;126(4):1198-1207.

87. Lopez-Verrilli MA, et al. Schwann cell-derived exosomes enhance axonal regeneration in the peripheral nervous system. Glia. 2013;61(11):1795-1806.

88. Natera-Naranjo O, et al. Identification and quantitative analyses of microRNAs located in the distal axons of sympathetic neurons. RNA. 2010;16(8):1516-1529.

89. Krichevsky AM, Gabriely G. miR-21: a small multi-faceted RNA.JCell MolMed.2009;13(1):39-53.

90. Ching RC, et al. Schwann cell-like differentiated adipose stem cells promote neurite outgrowth via secreted exosomes and RNA transfer. Stem Cell Res Ther. 2018;9(1):266.

91. Feliciano DM, et al. Embryonic cerebrospinal fluid nanovesicles carry evolutionarily conserved molecules and promote neural stem cell amplification. PLoS One. 2014;9(2):e88810.

92. Gong J, et al. Exosomes mediate cell contact- 
independent ephrin-Eph signaling during axon guidance. J Cell Biol. 2016;214(1):35-44.

93. Li T, et al. Activation of BDNF/TrkB pathway promotes prostate cancer progression via induction of epithelial-mesenchymal transition and anoikis resistance. FASEB J. 2020;34(7):9087-9101.

94. Au CW, et al. Tyrosine kinase $\mathrm{B}$ receptor and BDNF expression in ovarian cancers - Effect on cell migration, angiogenesis and clinical outcome. Cancer Lett. 2009;281(2):151-161.

95. Xu Z, et al. Tumor-derived exosomes educate fibroblasts to promote salivary adenoid cystic carcinoma metastasis via NGF-NTRK1 pathway. Oncol Lett. 2019;18(4):4082-4091.

96. Delpech JC, et al. Neuroimmune crosstalk through extracellular vesicles in health and disease. Trends Neurosci. 2019;42(5):361-372.

97. Maybruck BT, et al. Tumor-derived exosomes induce $\mathrm{CD} 8^{+} \mathrm{T}$ cell suppressors. JImmunother Cancer. 2017;5(1):65.

98. Chen G, et al. Exosomal PD-L1 contributes to immunosuppression and is associated with antiPD-1 response. Nature. 2018;560(7718):382-386.

99. Chen G, et al. PD-L1 inhibits acute and chronic pain by suppressing nociceptive neuron activity via PD-1. Nat Neurosci. 2017;20(7):917-926.

100.Simeoli R, et al. Exosomal cargo including microRNA regulates sensory neuron to macrophage communication after nerve trauma. Nat Commun. 2017;8(1):1778.

101. Chida Y, et al. Do stress-related psychosocial factors contribute to cancer incidence and survival? Nat Clin Pract Oncol. 2008;5(8):466-475.

102. Kelly WK, et al. Randomized, double-blind, placebo-controlled phase III trial comparing docetaxel and prednisone with or without bevacizumab in men with metastatic castration-resistant prostate cancer: CALGB 90401. JClin Oncol. 2012;30(13):1534-1540.

103. Coarfa C, et al. Influence of the neural microenvironment on prostate cancer. Prostate. 2018;78(2):128-139.

104.Brown MT, et al. Tanezumab reduces osteoarthritic hip pain: results of a randomized, double-blind, placebo-controlled phase III trial. Arthritis Rheum. 2013;65(7):1795-1803.

105. Cocco E, et al. NTRK fusion-positive cancers and TRK inhibitor therapy. Nat Rev Clin Oncol. 2018;15(12):731-747.

106.Doebele RC, et al. Entrectinib in patients with advanced or metastatic NTRK fusion-positive solid tumours: integrated analysis of three phase 1-2 trials. Lancet Oncol. 2020;21(2):271-282.

107. Hong DS, et al. Larotrectinib in patients with TRK fusion-positive solid tumours: a pooled analysis of three phase $1 / 2$ clinical trials. Lancet Oncol. 2020;21(4):531-540.

108. Lu H, et al. Impact of beta-blockers on prostate cancer mortality: a meta-analysis of 16,825 patients. Onco Targets Ther. 2015;8:985-990.

109. Yap A, et al. Effect of beta-blockers on cancer recurrence and survival: a meta-analysis of epidemiological and perioperative studies. $\mathrm{Br} \mathrm{J}$ Anaesth. 2018;121(1):45-57.

110. Jayaseelan VP. Emerging role of exosomes as promising diagnostic tool for cancer. Cancer Gene Ther. 2020;27(6):395-398.

111. Liang $\mathrm{G}$, et al. Engineered exosome-mediated delivery of functionally active miR-26a and its enhanced suppression effect in HepG2 cells. Int $J$ Nanomedicine. 2018;13:585-599.

112. Shi ZY, et al. Exosomal microRNAs-mediated intercellular communication and exosome-based cancer treatment. Int J Biol Macromol. 2020;158:530-541. 